\title{
L-Arginine/American Ginseng/Korean Ginseng/Ginkgo biloba-Based Supplement
}

National Cancer Institute

\section{Source}

Nationa/ Cancer Institute. L-Arginine/American Ginseng/Korean Ginseng/Ginkgo bilobaBased Supplement. NCI Thesaurus. Code C73130.

A proprietary nutritional supplement with potential male sexual function enhancement effect. L-Arginine/American ginseng/Korean ginseng/Ginkgo biloba-based supplement incorporates a standardized combination of Ginkgo biloba (24\% flavone glycosides, $6 \%$ terpene lactones), Korean ginseng (Panax ginseng -30\% ginsenosides), American ginseng (Panax quinquefolius - 5\% ginsenosides), L-Arginine, along with vitamins B6, B12, folate, thiamin, riboflavin, niacin, biotin, pantothenic acid, vitamins $A, C, E$, selenium, and zinc. L-Arginine is a precursor for nitric oxide (NO); ginseng may facilitate the conversion of L-Arginine into NO by NO synthase; the natural herb Ginkgo biloba may promote microvascular circulation. Overall, this supplement may increase NO production, which leads to vasodilatation and enhanced circulation. The improved blood flow is critical for sexual function and arousal. 